\title{
Implementação do Método de Monte Carlo em Avaliação de Risco Probabilís- tica Aplicada à Estimativa do Risco por Contato Dermal com Água Superficial Contaminada por Benzeno
}

\author{
Tirzah M. de Melo", Nelson O. Luna Caicedo* \\ tirzahmelo@hotmail.com e caicedo@iph.ufrgs.br
}

Recebido: 22/09/10 - revisado: 02/08/11 - aceito: 28/01/13

\section{RESUMO}

A condutividade hidráulica é o parâmetro hidrogeológico mais importante, influenciando fortemente os processos de fluxo e transporte de contaminantes no meio saturado. Para verificar as influências da variabilidade deste parâmetro sobre a estimativa do risco à saúde humana a metodologia de Avaliação de Risco Probabilística foi aplicada a um estudo de caso real utilizando-se do método estocástico mais amplamente utilizado no meio científico, o Método de Monte Carlo. Para tanto, a via de exposição por contato dermal com água superficial durante a natação foi considerada e o risco estocástico estimado. Os resultados da análise estocástica revelaram uma diferença de seis ordens de magnitude entre o valor mínimo e o máximo simulado e duas ordens de magnitude para o valor real do risco, indicando a dimensão das incertezas. A análise de sensibilidade do modelo demonstrou que a concentração do contaminante é o parâmetro de maior correlação com o risco, revelando a influência da condutividade hidráulica sobre as incertezas do modelo estocástico.

Palavras-chave: Método de Monte Carlo. Avaliação de risco probabilística. Estimativa estocástica do risco.

\section{INTRODUÇÃO}

Em 1983, o Conselho Nacional de Pesquisas dos Estados Unidos (National Research Council, NRC) lançou o relatório intitulado Avaliação de Risco no Governo Federal: Gerenciando o Processo (Risk Assessment in the Federal Government: Managing the Process), fornecendo a estrutura para que fossem conduzidas as avaliações de risco neste país (NATIONAL RESEARCH COUNCIL, 2007).

Tal processo consiste, segundo Kolluru (1996), na "estimativa da probabilidade de ocorrência de um evento indesejado e a provável magnitude de suas consequências durante um determinado periodo de tempo". Crawford-Brown (1999) ainda acrescenta que este é um processo puramente técnico, consistindo na aplicação de métodos que avaliem ou predigam o risco para situações específicas.

Atualmente, a metodologia mais utilizada para avaliação de risco à saúde humana foi desenvolvida pela ASTM (American Society of Testing and Materials), inicialmente elaborada para hidrocarbonetos e posteriormente estendida a outras substân-

*Instituto de Pesquisas Hidráulicas - UFRGS cias. Tal metodologia tem sido largamente aplicada e já aceita em muitos países como instrumento de legislação ambiental, denominando-se Risk Based Corrective Action (RBCA), traduzida como Ação Corretiva Baseada no Risco (TAKEUCHI; ROSEMBERG, 2009).

No escopo desta metodologia, tanto a variabilidade individual, espacial e temporal podem ser consideradas, bem como as incertezas dos dados que serão utilizados na estimativa do risco, os quais podem ser resultantes do emprego de modelos simplificados e conservadores, entre outros fatores.

A quantificação de tais incertezas lida com a estimativa de um nível de confiança no qual o risco esteja abaixo de um valor limite de aceitabilidade (SASSI; VERNAI; RUGGERI, 2007). Segundo National Research Council (1994) “(...) a análise de incertezas é o único meio de combater o 'falso senso de certeza', o qual é causado por uma rejeição para reconhecer e (tentar) quantificar a incerteza nas predições do risco".

Geralmente, em uma avaliação de risco à saúde humana, os parâmetros de entrada dos modelos são definidos em termos da estimativa mais provável, ignorando as incertezas associadas à utilização de um limitado número de dados para tais estimativas. Consequentemente, estes modelos não podem predizer ou estimar a incerteza do risco obtido, 
Implementação do Método de Monte Carlo em Avaliação de Risco Probabilística Aplicada à Estimativa do Risco por Contato Dermal com Água Superficial Contaminada por Benzeno

podendo influenciar na seleção da melhor alternativa de remediação (COPTY; FINDIKAKIS, 2000).

Em termos gerais, a incerteza representa a falta de conhecimento sobre fatores que afetam a exposição ou risco, enquanto a variabilidade emerge da heterogeneidade entre pessoas, lugares e tempo (variabilidade individual, espacial e temporal) (UNITED STATES ENVIRONMENTAL PROTECTION AGENCY, 1997). Isso implica que a incerteza conduz a estimativas imprecisas ou tendenciosas, enquanto a variabilidade pode afetar a precisão das estimativas e o grau para o qual elas podem ser generalizadas (SASSI; VERNAI; RUGGERI, 2007). Sander, Bergbäck e Öberg (2006) citam vários tipos de incertezas, tais como: erros de medidas, erro sistemático, imprecisão lingüística, julgamento subjetivo, variabilidade e aleatoriedade inerente.

A importância que começou a ser dada para esta abordagem estocástica resultou na criação de um novo ramo dentro da análise de risco, denominado avaliação de risco probabilística. Métodos inseridos neste contexto lidam com a variabilidade e as incertezas dos parâmetros nos modelos de avaliação de risco como sendo distribuições probabilísticas. $\mathrm{O}$ resultado deste processo é que o risco é estimado distribuindo-se em uma série de valores igualmente prováveis de ocorrer (MELO, 2010).

As fontes de incertezas relacionadas à avaliação de risco à saúde humana podem estar associadas a todas as etapas da metodologia, sob a forma de dados imprecisos, parâmetros mal atribuídos, modelos muito simplificados, etc.

Sua ampla aplicabilidade e habilidade de representar caso a caso os problemas de contaminação fizeram com que esta metodologia se tornasse o embasamento científico para o processo de tomada de decisões. Como resultado, evita-se que grandes quantias de recursos financeiros sejam empregadas visando alcançar padrões de remediação muitas vezes inatingíveis sob o ponto de vista tecnológico, o que justifica sua importância para a comunidade ambiental.

Tendo em vista a grande relevância atribuída aos aspectos estocásticos da avaliação de risco à saúde humana e suas implicações sobre o processo decisório no gerenciamento do risco, o presente artigo tem como objetivo apresentar a metodologia através de um estudo de caso real aplicado a uma situação de solo e água subterrânea contaminados em uma refinaria, utilizando-se do método estocástico mais amplamente utilizado no meio científico, o Método de Monte Carlo.

\section{AVALIAÇ̃̃O DE RISCO PROBABILÍSTICA}

Nesta abordagem estocástica, tipicamente determina-se um intervalo de confiança de $90 \%$ para o risco, variando de 5 (limite inferior de confiança) a 95\% (limite superior de confiança). Algumas das informações possíveis de serem obtidas quando se realiza uma avaliação de risco probabilística são:

- Propagação das incertezas inerentes aos dados e/ou aos modelos: permite que sejam determinados quais são os parâmetros ou fatores que realmente influenciam no valor do resultado final de estimativa por meio de uma análise de sensibilidade;

- Determinação da probabilidade de um risco ocorrer: uma vez que o risco é apresentado sob a forma de uma distribuição de probabilidades, então é possível determinar qual é a probabilidade de qualquer risco entre a faixa de valores correspondentes aos valores simulados ocorrer. Consequentemente, é possível determinar com que frequência um valor limite de risco aceitável é excedido ou igualado para que se avalie a dimensão do problema;

- Análise espacial e temporal das incertezas: Também é possível realizar análises da variabilidade espacial e/ou temporal de um parâmetro com o intuito de que se possam determinar regiões na área contaminada que necessitam de maior atenção no plano de remediação, bem como os períodos mais críticos de exposição passada, presente e/ou futura.

Os métodos empregados para propagação de incertezas envolvem tanto equações analíticas para modelos simples como aproximações numéricas para modelos mais complexos (HAMMONDS; HOFFMAN; BARTELL, 1994). Dentre os modelos analíticos pode-se citar o método de propagação da variância (variance propagation), o qual divide-se em modelo aditivo e modelo multiplicativo.

Dentre os modelos numéricos, o método de simulação de Monte Carlo (MC) se apresenta como o mais utilizado em avaliações de risco probabilísticas por ser mais simples e genérico. Este método foi inicialmente desenvolvido em 1940 e utiliza técnicas de amostragem estatística visando obter uma apro- 
ximação probabilística para a solução de uma equação matemática ou modelo (HAYSE, 2000).

Poulter (1998) ainda cita que o Método de Monte Carlo permite, em estágio mais complexo, avaliar a incerteza e variabilidade separadamente, sendo possível usar os resultados obtidos da simulação para selecionar tanto o segmento da população a ser protegida quanto o grau de confiança em que o desejado nível de proteção será atingido.

Outros métodos numéricos podem ser citados. Zhao et al. (2007) utilizaram em seu trabalho o método de simulação gaussiana seqüencial (SGS) para gerar a distribuição espacial da probabilidade das concentrações de $\mathrm{Cu}$ e $\mathrm{Pb}$ estarem acima de um determinado valor limite.

Sassi, Vernai e Ruggeri (2007) aplicaram a metodologia apresentada no Guia para Expressão da Incerteza em Medições (Guide to the Expression of Uncertainty in Measurement, GUM) para estimar as incertezas do risco à saúde humana e a contribuição de cada variável para estas incertezas. Avagliano e Parrella (2009) apresentam o método de análise de sensibilidade global (global sensitivity analysis, GSA) como um meio de auxiliar na investigação das fontes de incerteza e variabilidade dos modelos de avaliação da exposição por inalação de vapores.

Copty e Findikakis (2000) apresentam o método da aproximação de pequena perturbação (small pertubation approximation), no qual os parâmetros de fluxo, como condutividade hidráulica, são recolocados na equação de fluxo por sua média mais um termo da média gerado pela perturbação. Comparado ao método de Monte Carlo, o método citado apresenta a vantagem de menor esforço computacional, ao passo que está limitado a casos onde a variância dos termos da perturbação seja pequena.

O método de análise das probabilidades limitadas (probability bounds analysis, PBA) foi aplicado por Sander, Bergbäck e Öberg, (2006) na etapa de avaliação da exposição. Esta técnica é baseada na probabilidade em blocos (p-blocos) ao invés de apresentar os parâmetros de entrada dos modelos como distribuições probabilísticas. Dessa forma, cada bloco contém uma série de diferentes distribuições probabilísticas para cada parâmetro.

Outros autores foram ainda mais longe (van der VOET; SLOB, 2007), apresentando a integração entre as incertezas e variabilidade obtidas da etapa de avaliação da exposição com a etapa de caracterização do risco. Isso demonstra que qualquer etapa do processo de avaliação de risco à saúde humana pode ser abordada sob um ponto de vista probabilís- tico, incorporando as incertezas inerentes em cada uma delas.

Algumas limitações das aproximações probabilísticas, segundo Chowdhury, Champagne e McLellan (2009), são:

- Necessidade de um grande número de dados para gerar as distribuições dos parâmetros. A validade dos resultados irá depender da quantidade de informação empírica disponível ou do acerto das simplificações empregadas;

- Em geral, os métodos se baseiam em uma única distribuição de probabilidade de saída que agrega a variabilidade natural e inerente ao sistema às incertezas acerca da falta de conhecimento do fenômeno, impossibilitando mensurar a contribuição da incerteza que pode ser removida;

- Ao contrário dos métodos determinísticos, os métodos estocásticos não permitem a realização dos cálculos inversos (backward) da metodologia RBCA, os quais determinam as metas de remediação do sítio contaminado. Estes cálculos são de difícil realização e não podem ser obtidos por meio de simulação simples de Monte Carlo, exceto pelo uso de técnicas de tentativa e erro, devido à complexidade dos modelos envolvidos e, obviamente, que tal dificuldade também se aplica a modelos determinísticos complexos;

- Conforme o número de parâmetros do modelo avaliado aumenta, cada vez se torna mais difícil a obtenção da probabilidade de excedência. Isso implica que é praticamente impossível dimensionar toda a incerteza resultante do emprego de um modelo, uma vez que não é possível avaliar todas as suas fontes.

Embora muitos métodos de propagação das incertezas de um modelo se apliquem aos mais diversos casos, como apresentado, o método de Monte Carlo foi escolhido por ser mais simples e de fácil implementação computacional, sendo sua descrição apresentada no item seguinte.

\section{MÉTODO DE MONTE CARLO}

O método de Monte Carlo (MC) pode ser definido como um método computacional utilizado 
para gerar distribuições de probabilidades de uma variável dependente de outras variáveis que também são representadas por distribuições probabilísticas (POULTER, 1998). Desta forma, o valor médio provável de uma variável de interesse (abordagem determinística) é substituído por uma distribuição de valores dentro de uma faixa determinada por um conjunto de dados menor a partir do qual ela foi gerada (MELO, 2010).

O princípio do método consiste em amostrar aleatoriamente valores das distribuições dos parâmetros de interesse e aplicá-los no modelo que se deseja propagar as incertezas. Isto é feito, em geral, milhares de vezes, selecionando-se milhares de combinações possíveis (igualmente prováveis de ocorrerem) dos valores dos parâmetros. Com isso, o resultado de saída (no presente caso, o risco) é apresentado sob a forma de uma distribuição probabilística que tem tantos valores quanto for o número de simulações ou iterações de MC (MELO, 2010).

$\mathrm{Na}$ sua forma mais simples, o método de Monte Carlo necessita apenas de informações sobre a natureza das distribuições dos parâmetros de interesse e seus atributos característicos (média, desviopadrão, moda, máximo, mínimo, etc.), resultando numa análise pontual das incertezas. No entanto, como citado anteriormente, também é possível realizar a mesma análise, porém incorporando aspectos como a heterogeneidade espacial de algum parâmetro ou sua variabilidade temporal, o que exige um aparato muito maior de informações (MELO, 2010).

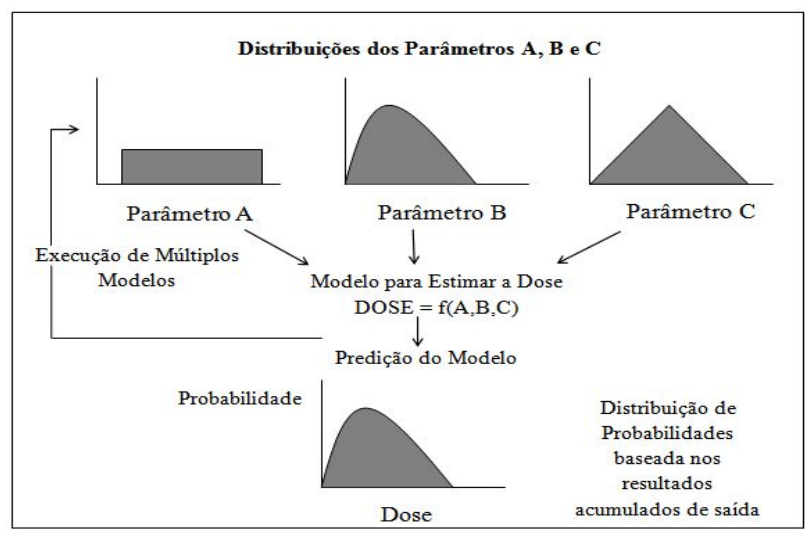

Figura 1 - Representação esquemática do método de Monte Carlo (Adaptado de HAYSE, 2000).

O método de Monte Carlo pode ser executado por meio de dois métodos de amostragem aleatória: Amostragem Aleatória Simples (Simple Random Sampling, SRS) e Amostragem do Hipercubo Latino (Latin Hypercube Sampling, LHS). No primeiro caso
(Figura 1), um valor aleatório é amostrado de cada distribuição especificada para cada parâmetro do modelo, e um valor de saída é obtido, sendo o processo repetido para um número especificado de simulações de Monte Carlo. No outro método, a distribuição de cada parâmetro é seccionada em faixas de igual probabilidade, sendo que o número de secções se iguala ao número de simulações a serem feitas (HAMMONDS; HOFFMAN; BARTELL, 1994). De cada secção são amostrados aleatoriamente valores do parâmetro considerado, sem reposição.

\section{ESTUDO DE CASO}

Dentro de um contexto hidrogeológico, especificamente contaminação de água subterrânea, a importância de avaliar o risco sob um aspecto estocástico está diretamente associada à descontinuidade espacial da condutividade hidráulica (K) (entre outros parâmetros como porosidade, transmissividade, etc.), a qual influencia fortemente os processos de fluxo e transporte de contaminantes no meio saturado. Desconsiderar esta característica intrínseca de heterogeneidade do meio físico implica em estimativas pouco confiáveis do risco.

A aplicação de grande parte dos conceitos expostos anteriormente foi feita através de uma análise estocástica da equação do risco para efeitos carcinogênicos, considerando a via de exposição por contato dermal com água superficial durante a natação, contaminada por benzeno a partir da água subterrânea.

A área de estudo refere-se ao antigo depósito de resíduos oleosos de uma refinaria localizada na região Sudeste do país, cuja identificação não será divulgada por motivos contratuais.

A via de exposição mencionada foi aqui preferencialmente escolhida devido à presença de pessoas que, durante o verão, se banham em um córrego localizado a $200 \mathrm{~m}$ do depósito de resíduos oleosos na refinaria.

Desta forma, o método de Monte Carlo foi aplicado ao modelo de transporte de contaminantes em água subterrânea. Para avaliar o risco, considerou-se que o aporte de contaminante que chega ao córrego é igual à concentração do mesmo que é transportado da fonte até o córrego através do fluxo subterrâneo.

A equação que descreve o risco para efeitos carcinogênicos de exposição por contato dermal é dada por (UNITED STATES ENVIRONMENTAL PROTECTION AGENCY, 1989): 


$$
\mathrm{R}=\frac{\mathrm{C} \times \mathrm{AS} \times \mathrm{PC} \times \mathrm{ET} \times \mathrm{EF} \times \mathrm{ED} \times \mathrm{CF}}{\mathrm{BW} \times \mathrm{AT}} \times \mathrm{SF}
$$

Onde: $\quad$ AS = área da superfície da pele disponível para contato $\left(\mathrm{cm}^{2}\right)$;

$\mathrm{PC}=$ constante químico-específica de permeabilidade dermal $(\mathrm{cm} / \mathrm{h})$; $(\mathrm{h} / \mathrm{d})$;

ET = tempo duração do evento (banho)

$\mathrm{EF}=$ frequência da exposição (d/ano);

$\mathrm{ED}=$ duração da exposição (anos);

$\mathrm{CF}$ = fator de conversão volumétrico para a água $\left(1 \mathrm{~L} / 1000 \mathrm{~cm}^{3}\right)$;

$\mathrm{SF}=$ fator de carcinogenicidade do contaminante para contato dermal (kg.d $/ \mathrm{mg})$;

$\mathrm{BW}=$ massa corpórea $(\mathrm{kg})$;

$\mathrm{AT}=$ tempo médio de avaliação da exposição (dias);

$\mathrm{C}=$ concentração do contaminante no ponto de exposição $(\mathrm{mg} / \mathrm{L})$, dado por:

$C=C(x, y, z, t)=\frac{M_{2}}{\varphi_{e} R} f_{1}(x, t) \cdot F_{2}(y, t) \cdot F_{3}(z, t)$

$$
\begin{aligned}
f_{1}(x, t)= & \frac{1}{2\left(\pi D_{x} t / R\right)^{1 / 2}} . \\
& \exp \left\{\left[-(x-(v t / R))^{2} /\left(4 D_{x} t / R\right)\right\}\right.
\end{aligned}
$$

$$
\begin{aligned}
\mathrm{F}_{2}(\mathrm{y}, \mathrm{t})=\frac{1}{2}\left\{\operatorname{erf}\left[(\mathrm{y}+\mathrm{Y}) /\left(2\left(\mathrm{D}_{\mathrm{y}} \mathrm{t} / \mathrm{R}\right)^{1 / 2}\right)\right]\right. \\
-\operatorname{erf}\left[(\mathrm{y}-\mathrm{Y}) /\left(2\left(\mathrm{D}_{\mathrm{y}} \mathrm{t} / \mathrm{R}\right)^{1 / 2}\right]\right\}
\end{aligned}
$$

$$
\begin{aligned}
\mathrm{F}_{3}(\mathrm{z}, \mathrm{t})=\frac{1}{2}\left\{\operatorname{erf}\left[(\mathrm{z}+\mathrm{Z}) /\left(2\left(\mathrm{D}_{\mathrm{z}} \mathrm{t} / \mathrm{R}\right)^{1 / 2}\right)\right]\right. \\
-\operatorname{erf}\left[(\mathrm{z}-\mathrm{Z}) /\left(2\left(\mathrm{D}_{\mathrm{z}} \mathrm{t} / \mathrm{R}\right)^{1 / 2}\right]\right\}
\end{aligned}
$$

Para determinação da distribuição probabilística da concentração de benzeno partiu-se das seguintes relações entre outros parâmetros:

$$
\mathrm{M}_{2}=\mathrm{C}_{\mathrm{o}} \times \mathrm{q}
$$

$\mathrm{q}=\mathrm{K} . \mathrm{i}$ $\mathrm{v}=\frac{\mathrm{q}}{\phi_{\mathrm{e}}}$

Onde: $\quad \mathrm{q}=$ velocidade de $\operatorname{Darcy}(\mathrm{L} / \mathrm{T})$;

$\mathrm{i}=$ gradiente hidráulico $(\mathrm{L} / \mathrm{L})$;

$\mathrm{K}$ = condutividade hidráulica saturada $(\mathrm{L} / \mathrm{T})$;

$\mathrm{v}=$ velocidade de percolação $(\mathrm{L} / \mathrm{T})$;

$\mathrm{M}_{2}=$ massa liberada por unidade de área para $\mathrm{o}$ aquífero $\left(\mathrm{M} / \mathrm{L}^{2} . \mathrm{T}\right)$;

$\phi_{\mathrm{e}}=$ porosidade efetiva do aquífero;

$\mathrm{C}_{\mathrm{o}}=$ concentração do contaminante em água subterrânea na fonte $\left(\mathrm{M} / \mathrm{L}^{3}\right)$.

As equações (2) a (8) referem-se às notas de aula da disciplina AE-638 Groundwater Quality and Contaminant Transport do Professor David B. McWhorter (1995), da Universidade do Estado do Colorado, EUA (Colorado State University, USA) (MELO, 2010).

Desta forma, a metodologia de propagação das incertezas sobre o modelo da equação foi dividida em duas partes devido à complexidade da equação (2), compreendendo as etapas descritas abaixo:

1. Propagação das incertezas sobre o modelo de transporte de contaminantes: Nesta etapa, o modelo para transporte de contaminantes passando através de um plano normal ao fluxo subterrâneo (equações 2 a 8) foi utilizado, sendo que a variável considerada na propagação das incertezas foi a condutividade hidráulica;

2. Propagacãa das incertezas sobre o modelo de risco à saúde humana para efeitos carcinogênicos: Os resultados obtidos na etapa 1 (distribuição probabilística de C) foram então aplicados na equação 1, juntamente com as distribuições dos demais parâmetros desta equação para obtenção da distribuição de probabilidades do risco.

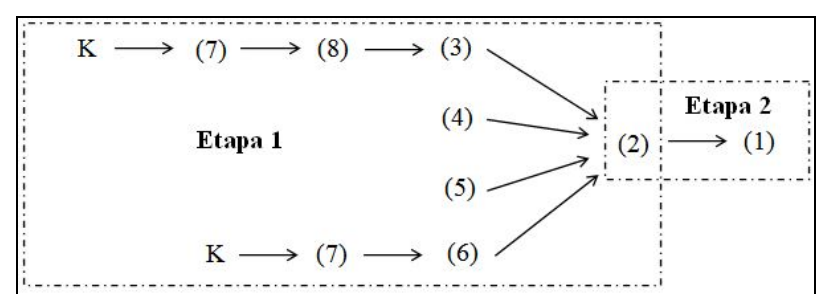

Figura 2 - Esquema ilustrativo das simulações estocásticas

Assim, para ambas as etapas acima foi aplicado o método de Monte Carlo por meio da geração de números aleatórios que representem as distribuições escolhidas para os parâmetros de interesse. A 
figura 2 apresenta um esquema ilustrativo das etapas 1 e 2 descritas acima.

\section{ANÁLISE DOS DADOS DE CONDUTIVIDADE HIDRÁULICA}

Os valores amostrados para a condutividade hidráulica foram utilizados para simular o transporte de contaminantes em água subterrânea contaminada para determinar a concentração de contaminante que chega ao córrego e posteriormente determinar o risco. Os valores de K estão apresentados na tabela 1, de onde é possível verificar a grande variação na área da refinaria, bem como a pequena magnitude dos mesmos, indicando a baixa capacidade de movimentação da água subterrânea.

Para verificar se os dados de $\mathrm{K}$ ou $\ln (\mathrm{K})$ estão normalmente distribuídos, o teste de aderência de Lilliefors foi aplicado, indicando que se pode assumir a normalidade para os valores de $\ln (\mathrm{K})$ com um nível de significância de $5 \%$. Este teste é similar ao teste de Kolmogorov-Smirnov. No entanto, ele testa um conjunto de dados apenas para a normalidade, enquanto o outro testa para qualquer distribuição (ARAÚJO et al., 2010).

Tabela 1 - Dados de condutividade hidráulica amostrados na área da refinaria

\begin{tabular}{ccc}
\hline Amostra & K (m/dia) & $\begin{array}{c}\text { Valores Padroniza- } \\
\text { dos de K }\end{array}$ \\
\hline 1 & 0,90207 & 1,53259 \\
2 & 0,37479 & 0,10558 \\
3 & 1,37311 & 2,80729 \\
4 & 0,34944 & 0,03697 \\
5 & 0,46306 & 0,34447 \\
6 & 0,23545 & $-0,27153$ \\
7 & 0,31777 & $-0,04874$ \\
8 & 0,09753 & $-0,64479$ \\
9 & 0,46696 & 0,35502 \\
10 & 0,10391 & $-0,62752$ \\
11 & 0,12640 & $-0,56666$ \\
12 & 0,08489 & $-0,67900$ \\
13 & 0,08392 & $-0,68162$ \\
14 & 0,01866 & $-0,85824$ \\
15 & 0,03868 & $-0,80406$ \\
\hline
\end{tabular}

Fonte: Não divulgada.
Os valores de $\ln (\mathrm{K})$ foram ajustados por uma distribuição normal com parâmetros $\mu_{\operatorname{lnK}}=-1,65$ $(\ln (\mathrm{m} /$ dia $))$ e $\sigma_{\operatorname{lnK}}=1,17(\ln (\mathrm{m} /$ dia $))$. Os gráficos na figura 3 permitem uma inspeção visual da qualidade do ajuste segundo uma distribuição normal para os logaritmos de K.

Uma vez identificada a forma da distribuição de $\operatorname{lnK}$ e seus parâmetros, estas informações foram inseridas no programa SEC (Simulações Estocástica da Concentração) desenvolvido em linguagem do MATLAB ${ }^{\circledR}$ especificamente para este estudo de caso, para o qual foram utilizados os dados para simulação da concentração do benzeno apresentados na tabela 2.

Foram realizadas 5.000 simulações de Monte Carlo, implicando, igualmente, na geração de 5.000 valores aleatórios de $q, v$ e $C$.

$\mathrm{Na}$ figura 4 está apresentado o histograma de frequências dos 5.000 valores de $\mathrm{C}$ de equilíbrio ( $\mathrm{mg} / \mathrm{L})$ obtidos, por meio do qual é possível observar um comportamento aproximadamente lognormal para os dados. Também está representado nesta figura o histograma de frequências dos 5.000 valores aleatórios de $\mathrm{K}$ que geraram os valores de $\mathrm{C}$, o qual demonstra que tais valores de $\mathrm{K}$ seguem uma distribuição log-normal, como desejado.
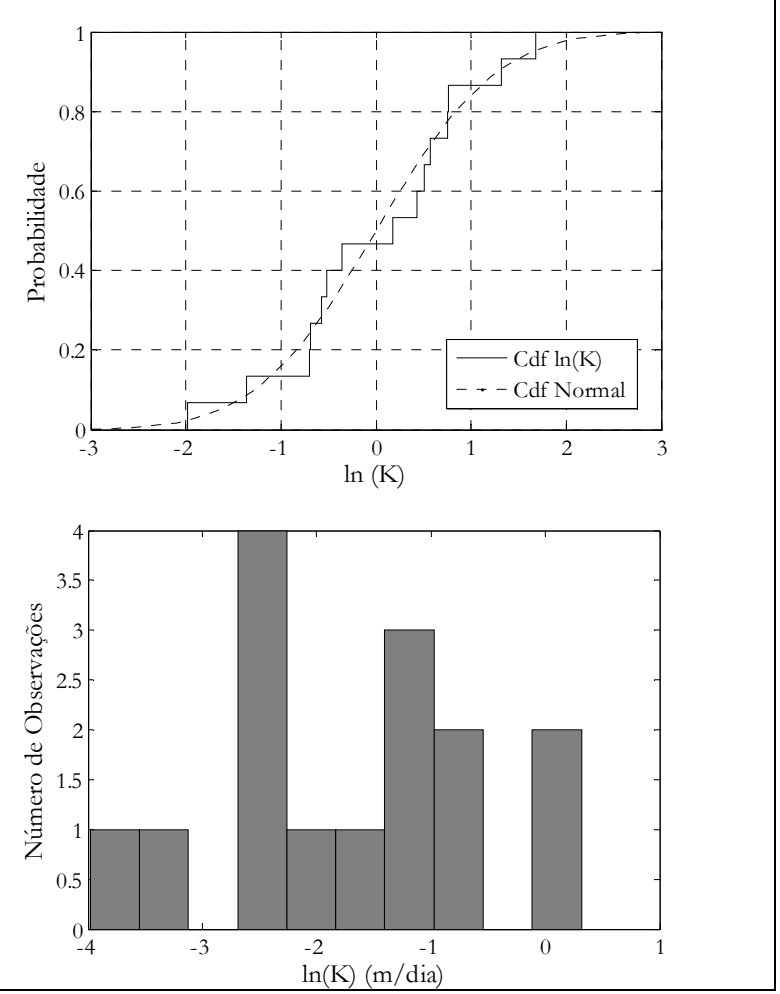

Figura 3 - Inspeção visual da normalidade para os valores de $\ln K$ 
Tabela 2 - Dados utilizados para a simulação da concentração do benzeno

\begin{tabular}{lll}
\hline Parâmetro & Valor & Descrição \\
\hline$(\mathrm{x}, \mathrm{y}, \mathrm{z})(\mathrm{m})$ & $(200,0,0)$ & Ponto de exposição do receptor. \\
$\mathrm{t}(\mathrm{dias})^{*}$ & 70 anos & Tempo de simulação após o início da contaminação. \\
$\alpha_{\mathrm{x}}(\mathrm{m})$ & 40 & Dispersividade longitudinal estimada pela equação. \\
$\alpha_{\mathrm{y}}(\mathrm{m})$ & 13 & Dispersividade transversal estimada pela equação. \\
$\alpha_{\mathrm{z}}(\mathrm{m})$ & 2 & Dispersividade transversal estimada pela equação. \\
$\mathrm{D}_{\mathrm{x}}\left(\mathrm{m}^{2} / \mathrm{dia}\right)$ & 0,26 & Coeficiente de dispersão longitudinal estimado por $\mathrm{D}_{\mathrm{x}}=\mathrm{v} . \alpha_{\mathrm{x} .}$ \\
$\mathrm{D}_{\mathrm{y}}\left(\mathrm{m}^{2} / \mathrm{dia}\right)$ & 0,08 & Coeficiente de dispersão transversal estimado por $\mathrm{D}_{\mathrm{y}}=\mathrm{v} . \alpha_{\mathrm{y} .}$ \\
$\mathrm{D}_{\mathrm{z}}\left(\mathrm{m}^{2} / \mathrm{dia}\right)$ & 0,01 & Coeficiente de dispersão transversal estimado por $\mathrm{D}_{\mathrm{z}}=\mathrm{v} . \alpha_{\mathrm{z} .}$. \\
$\mathrm{v}(\mathrm{m} / \mathrm{ano})$ & 2,336 & Velocidade do fluxo subterrâneo estimado por $\mathrm{v}=\mathrm{q} / \phi$. \\
$\mathrm{q}(\mathrm{m} / \mathrm{ano})$ & 0,438 & Descarga específica do aqüífero ou velocidade de $\mathrm{Darcy}$. \\
$\phi(-)$ & 0,19 & Porosidade efetiva medida no campo. \\
$\mathrm{Y}(\mathrm{m})$ & 10 & Largura da pluma na fonte medida do mapa. \\
$\mathrm{Z}(\mathrm{m})$ & 3 & Espessura da pluma na fonte. \\
$\mathrm{R}(-)$ & 1 & Retardo do contaminante. \\
$\mu(1 / \mathrm{ano})$ & 0 & Sem decaimento de primeira ordem do benzeno. \\
$\mathrm{C}_{\mathrm{o}}(\mathrm{mg} / \mathrm{L})$ & 0,0019 & Concentração de benzeno em água subterrânea. \\
\hline
\end{tabular}

Fonte: não divulgada. ${ }^{*}$ Tempo necessário para que se atinja a concentração de equilíbrio do benzeno.

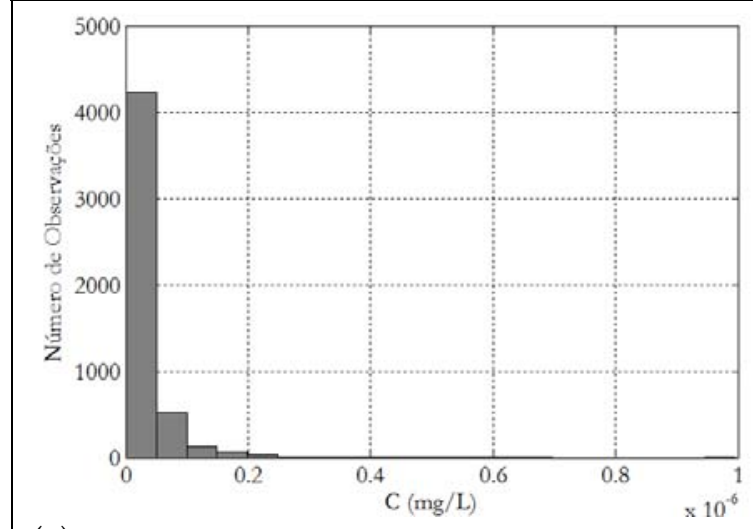

(a)

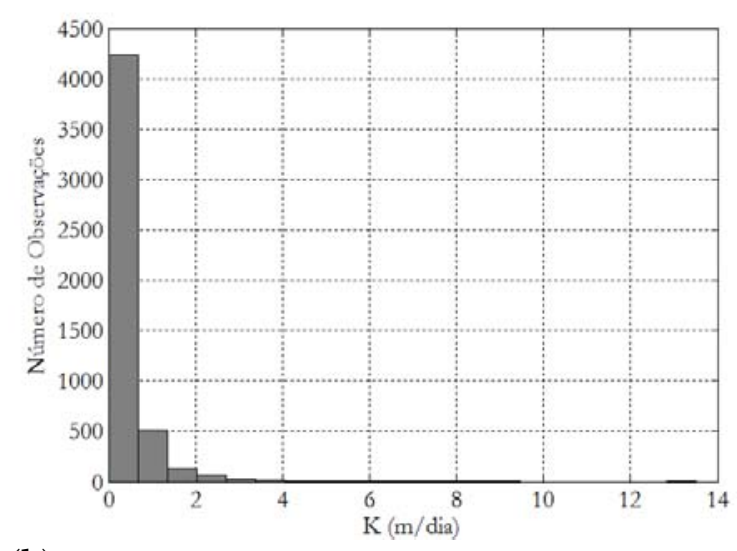

(b)

Figura 4 - Histograma das 5.000 simulações de $\mathrm{C}$ de equilíbrio em (a) e dos 5.000 valores aleatórios de K em (b)
No entanto, a fim de que se obtivesse o melhor ajuste, foram testadas (ajustadas) várias distribuições para $\mathrm{C}$ através da ferramenta dfittool do MATLAB ${ }^{\circledast}$. Esta informação é indispensável, pois necessita-se saber a forma e os parâmetros da distribuição de $\mathrm{C}$ para que esta alimente a etapa 2 da metodologia. Os melhores ajustes ocorreram para as distribuições log-normal e log-logística. Escolheu-se a primeira, pois esta já foi citada por Lemke e Bahrou (2009) para caracterizar C; tendo como parâmetros: $\mu=-18,0316(\mathrm{mg} / \mathrm{L})$ e $\sigma=$ $1,1818(\mathrm{mg} / \mathrm{L})$.

\section{SIMULAÇÃO ESTOCÁSTICA DO RISCO À SAÚDE HUMANA}

O item anterior descreveu uma etapa importante e necessária para a propagação das incertezas na predição do risco, cujo principal objetivo era a determinação da forma e dos parâmetros da distribuição de probabilidades da concentração de benzeno em água subterrânea que chega ao córrego.

Desta forma, dada a Equação (1) e as informações descritas na tabela 3 para as variáveis de entrada do modelo do risco, foram realizadas 65.000 iterações para a simulação estocástica. Para tanto, o programa Simulación 4.0 (VARELA, 2003) foi utilizado, o qual gera $\mathrm{N}$ combinações possíveis das 
Implementação do Método de Monte Carlo em Avaliação de Risco Probabilística Aplicada à Estimativa do Risco por Contato Dermal com Água Superficial Contaminada por Benzeno

Tabela 3 - Parâmetros e distribuições das variáveis para a simulação de Monte Carlo do risco à saúde humana por contato dermal com água superficial

\begin{tabular}{|c|c|c|c|}
\hline Parâmetro & Descrição & Unidades & Tipo de Distribuição \\
\hline $\mathrm{C}$ & Concentração de benzeno & $\mathrm{mg} / \mathrm{L}$ & Log-normal $^{\text {a }} ; \mu=-18,0316$ e $\sigma=1,1818$ \\
\hline $\mathrm{EF}$ & Frequência de exposição & dias/ano & Triangular $^{\mathrm{b}} ; 100 / 300 / 365$ \\
\hline ED & Duração da exposição & anos & Triangular $^{\mathrm{b}} ; 1 / 10 / 70$ \\
\hline BW & Massa corpórea & $\mathrm{kg}$ & Normal $^{\mathrm{b}} ; \mu=71$ e $\sigma=15,9$ \\
\hline AT & Tempo de exposição média & dias & Triangular ${ }^{\mathrm{b}} ; 30 / 80 / 90$ \\
\hline $\mathrm{SF}_{\mathrm{d}}$ & Fator de carcinogenicidade & kg.dia/mg & Constante $^{c} ; 0,0299$ \\
\hline $\mathrm{PC}$ & Permeabilidade da pele & $\mathrm{m} /$ hora & Uniforme $^{\mathrm{b}} ;$ mín $=0,004$, máx $=0,01$ \\
\hline AS & Área da superfície da pele & $\mathrm{m}^{2}$ & Constante $^{c} ; 2,3$ \\
\hline $\mathrm{CF}$ & Fator de conversão & $\mathrm{L} / \mathrm{m}^{3}$ & Constante $^{\mathrm{b}} ; 1000$ \\
\hline ET & Tempo do evento (banho) & horas & Uniforme $^{\mathrm{d}} ;$ mín $=1$, máx $=4$ \\
\hline
\end{tabular}

${ }^{\mathrm{a}}$ Simulada e ajustada no item anterior; ${ }^{\mathrm{b}}$ Lemke e Bahrou (2009); ${ }^{\mathrm{c}}$ sugeridos no Software RBCA Tool Kit for Chemical Releases, Versão 1.3a; ${ }^{\mathrm{d}}$ Suposto.

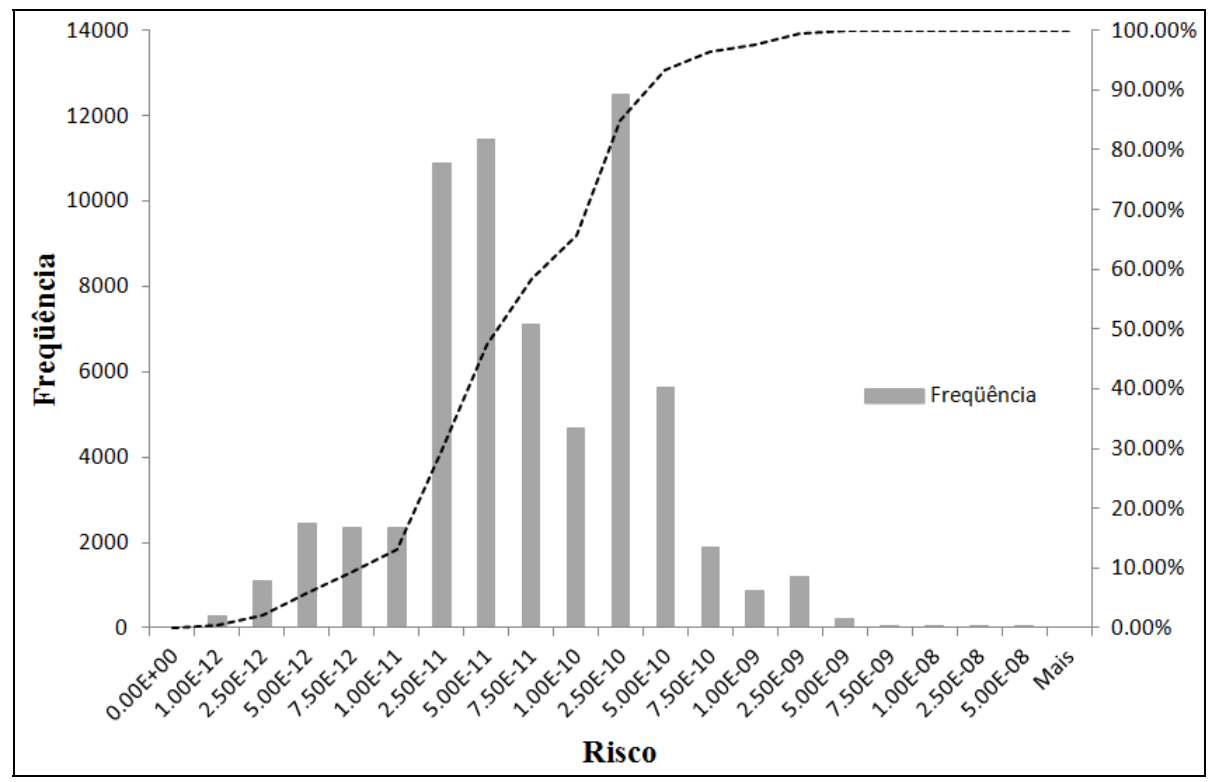

Figura 5 - Histograma de frequências e de frequências cumulativas do risco à saúde humana

variáveis de entrada obtidas a partir das distribuições probabilísticas a elas atribuídas.

Embora não tenha sido considerado no presente estudo de caso, no referido programa também é possível abordar as correlações existentes entre qualquer uma das variáveis. Este fato é muito importante, pois a simulações para uma determinada variável são realizadas segundo a correlação existente com outras variáveis, afetando a estimativa do risco.

O sumário estatístico para as variáveis descritas na tabela 3 bem como o risco simulado estão apresentados na tabela 4 , de onde é possível verifi- car que a incerteza sobre o valor real do risco compreende uma faixa de 6 ordens de magnitude para a simulação estocástica realizada.

Na figura 5 estão apresentados o histograma de frequências e de frequências acumuladas para os valores de risco obtidos pela simulação estocástica. Admitiu-se um risco limite aceitável de 1,0E-06, para o qual observa-se que em $100 \%$ das simulações ele não foi excedido devido a fatores como baixas condutividade hidráulica, velocidade de percolação e concentração no ponto de exposição. 
Este argumento poderia ser melhor compreendido caso o risco limite aceitável pertencesse à faixa de valores simulados, indicando qual a probabilidade dele ser excedido, informação esta que seria de extrema importância para o desenvolvimento de um plano de remediação. Na figura 5 também é possível constatar que quase $100 \%$ das simulações são menores do que 3,00E-09.

Tabela 4 - Sumário estatístico para as variáveis do modelo do risco correspondente às 65.000 simulações

\begin{tabular}{lllll}
\hline Parâmetro & Máximo & Mínimo & Média & Variância \\
\hline RISCO & $2,86 \mathrm{E}-08$ & $9,16 \mathrm{E}-14$ & $1,59 \mathrm{E}-10$ & $1,74 \mathrm{E}-19$ \\
AT & 89,88 & 30,18 & 66,70 & 171,83 \\
BW & 138,10 & 3,83 & 71,03 & 254,28 \\
C & $1,89 \mathrm{E}-06$ & $7,94 \mathrm{E}-11$ & $2,96 \mathrm{E}-08$ & $2,49 \mathrm{E}-15$ \\
ED & 69,95 & 1,10 & 26,93 & 234,21 \\
EF & 364,59 & 100,81 & 255,02 & 3183,60 \\
ET & 3,99 & 1,00 & 2,50 & 0,76 \\
PC & $9,99 \mathrm{E}-03$ & $4,00 \mathrm{E}-03$ & $7,02 \mathrm{E}-03$ & $2,99 \mathrm{E}-06$ \\
\hline
\end{tabular}

Também utilizando-se do programa Simulación 4.0 (VARELA, 2003), o resultado da análise de sensibilidade sobre as variáveis do modelo estocástico para simulação do risco à saúde humana está apresentado na figura 6. Neste gráfico, variáveis com baixo coeficiente de correlação podem ser consideradas independentes ou constantes em uma próxima simulação para o modelo do risco para contato dermal com água superficial durante a natação, pois interferem muito pouco no seu valor (ex.: AT, EF, PC e BW).

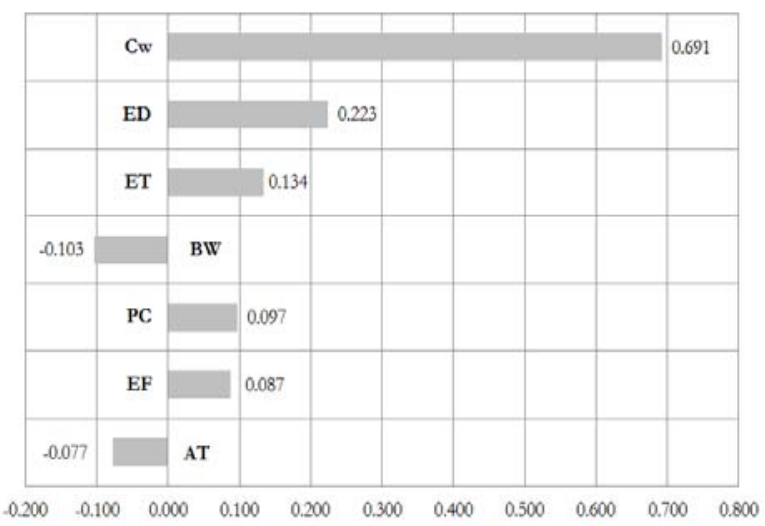

Figura 6 - Gráfico de correlação para as variáveis de entrada do modelo
O grande interesse de realizar uma avaliação de risco probabilística é quantificar as incertezas que podem ser mensuradas, por meio dos percentis de 5 e $95 \%$. Para o presente estudo de caso, os percentis correspondentes foram: 4,45E-12 a $6,02 \mathrm{E}-10$, indicando que o valor verdadeiro para o risco está situado entre esta faixa de valores com uma diferença de duas ordens de grandeza. Variação significativa, caso a distribuição de valores do risco fosse próximo de um valor limite aceitável.

\section{CONCLUSÕES}

O presente artigo teve como objetivo aplicar a metodologia de avaliação de risco probabilística a um estudo de caso real de contaminação de água subterrânea na área de um antigo depósito de resíduos oleosos em uma refinaria, partindo do pressuposto de que a condutividade hidráulica afetaria a estimativa do risco, da qual depende da concentração do contaminante. Assumiu-se que a contaminação da água superficial era alimentada pela água subterrânea contaminada desde a fonte na refinaria.

O método de Monte Carlo foi aplicado em duas etapas do trabalho: na geração de possíveis valores de concentrações de benzeno que chegam ao córrego advindos da água subterrânea, bem como na estimativa da distribuição de probabilidades do risco à saúde humana. Estes resultados foram obtidos a partir de valores aleatórios de condutividade hidráulica ajustados a uma distribuição probabilística com base nos dados amostrados na área de estudo.

Desta forma, a análise de sensibilidade indicou uma correlação muito grande do risco com a variável $\mathrm{C}$, a qual, por sua vez, foi modelada pela simulação estocástica da condutividade hidráulica. Logo, pode-se concluir que a condutividade hidráulica afeta a estimativa do risco, para o qual a análise estocástica revelou uma diferença de seis ordens de magnitude entre o valor mínimo e o máximo simulado e duas ordens de magnitude para o valor real do risco dentre de um intervalo de confiança.

Com base nos resultados supracitados, pode-se concluir que nem mesmo para as situações de máxima exposição, isto é, quando considera-se a concentração de estado de equilíbrio, não foram detectados riscos de efeitos carcinogênicos pelo benzeno pela via de exposição por contato dermal com água superficial durante a natação. Isto equi- 
Implementação do Método de Monte Carlo em Avaliação de Risco Probabilística Aplicada à Estimativa do Risco por Contato Dermal com Água Superficial Contaminada por Benzeno

vale dizer que, uma vez que se esteja considerando a concentração de equilíbrio na estimativa do risco, se está estimando o máximo risco possível de ocorrer.

A simulação estocástica realizada por meio do método de Monte Carlo expôs as incertezas propagadas das variáveis do modelo para o risco. Tal fato demonstra a importância de que estas incertezas sejam mensuradas, principalmente quando se lidam com concentrações altas de contaminantes, as quais podem resultar em risco de efeitos adversos à saúde humana.

Adicionalmente, a abordagem probabilística também permite detectar se uma estimativa determinística do risco está sub ou superestimando o valor verdadeiro, ou os prováveis valores verdadeiros do risco, refletindo a sua contribuição em avaliação de risco à saúde humana.

\section{REFERÊNCIAS}

ARAÚJO, E. M.; SILVA, I. N.; OLIVEIRA, J. B.; CAVALCANTE JR., E. G.; ALMEIDA, B. M. Aplicação de seis distribuições de probabilidade a séries de temperatura máxima em Iguatu - CE. Revista Ciência Agronômica, v. 41, n. 1, p. 36-45, 2010.

AVAGLIANO, S.; PARELLA, L. Managing uncertainty in risk-based corrective action design: global sensitivity analysis of contaminant fate and exposure models used in the dose assessment. Environmental Modeling $\mathcal{E}$ Assessment, v. 14, n. 1, p. 47-57, 2009.

CHOWDHURY, S.; CHAMPAGNE, P.; McLELLAN, P. J. Uncertainty characterization approaches for risk assessment of DPBs in drinking water: A review. Journal of Environmental Management, v. 90, p. 1680-1691, 2009.

COPTY, N. K.; FINDIKAKIS, A. N. Quantitative estimates of the uncertainty in the evaluation of ground water remediation schemes. Ground Water, v. 38, n. 1, p. 29-37, 2000.

CRAWFORD-BROWN, D. J. Risk-based environmental decisions: methods and culture. Norwell: Kluwer Academy, 1999. 224 p.

HAMMONDS, J. S.; HOFFMAN, F. O.; BARTELL, S. M. An introductory guide to uncertainty analysis in environmental and health risk assessment. Oak Ridge, Tenessee: [s.n.], 1994.

HAYSE, J. W. Using Monte Carlo analysis in ecological risk assessments. DuPage Conty: Argonne National Laboratory, 2000.

KOLLURU, R. V. Health risk assessment: principles and practices. In: KOLLURU, R. V.; BARTELL, S. M.; PITBLADO, R. M.; STRICOFF, R. S. (Ed.). Risk assessment and management handbook for environmental, health and safety professionals. New York: Mc-Graw Hill, 1996. p. 4.3-4.68.

LEMKE, L. D.; BAHROU, A. S. Partitioned multiobjective risk modeling of carcinogenic compounds in groundwater. Stochastic Environmental Research and Risk Assessment, v. 23, n. 1, p. 27-39, 2009.

McWHORTER, D. B. Notas de aula. Disciplina AE638: groundwater Quality and Contaminant Transport. Colorado: Colorado State University, 1995.

MELO, T. M. Soluções analíticas para transporte de hidrocarbonetos de petróleo em água subterrânea: avaliação determinística e probabilística do risco à saúde humana. 2010. 124 f. Dissertação (Mestrado) Universidade Federal do Rio Grande do Sul, Porto Alegre, 2010.

NATIONAL RESEARCH COUNCIL. Commission on Life Sciences. Board on Environmental Studies and Toxicology. Committee on Risk Assessment of Hazardous Air Pollutants. Science and judgment in risk assessment. Washington: National Academy Press, 1994. $651 \mathrm{p}$.

NATIONAL RESEARCH COUNCIL. Commission on Life Sciences. Board on Environmental Studies and Toxicology. Committee to Review the OMB Risk Assessment Bulletin. Scientific Review of the Proposed Risk Assessment Bulletin from the Office of Management and Budget. Washington: National Academy Press, 2007. 286 p.

POULTER, S. R. Monte Carlo Simulation in Environmental Risk Assessment - Science, Policy and Legal Issues. Risk: Health, Safety \& Environment, v. 7, p. 7-26, 1998.

SANDER, P.; BERGBÄCK, B.; ÖBERG, T. Uncertain numbers and uncertainty in the selection of input distri- 
butions - consequences for a probabilistic risk assessment of contaminated land. Risk Analysis, v. 26, n. 5, p. 1363-1375, 2006.

SASSI, G.; VERNAI, A. M.; RUGGERI, B. Quantitative estimation of uncertainty in human risk analysis. Journal of Hazardous Materials, v. 145, n. 1-2, p. 296-304, 2007.

TAKEUCHI, E.; ROSENBERG, A. Definição de riscos aceitáveis baseados no risco para hidrocarbonetos no Município de Porto Alegre segundo a metodologia RBCA. Revista Engenharia Sanitária $e$ Ambiental, v. 14, n. 4, p. 479-488, 2009.

UNITED STATES ENVIRONMENTAL PROTECTION AGENCY. USEPA. Guiding principles for Monte Carlo Analysis, EPA/630/R-97/001. [S.1.]: USEPA, 1997.

UNITED STATES ENVIRONMENTAL PROTECTION AGENCY. USEPA. Risk Assessment Guidance for Superfund. Volume I - Human Health Evaluation Manual (Parte A). [S.1.]: USEPA, 1989.

VAN DER VOET, H.; SLOB, W. Integration of Probabilistic Exposure Assessment and Probabilistic Hazard Characterization. Risk Analysis, v. 27, n. 2, p. 351-371, 2007.

VARELA, J. R. Simulación 4.0. Buenos Aires: Universidade del CEMA, 2003.

ZHAO, Y.; XU, X.; HUANG, B.; SUN, W.; SHAO, $\mathrm{X}$.; SHI, X.; RUAN, X. Using robust kriging and sequential Gaussian simulation to delineate the copper- and lead- contaminated areas of a rapidly industrialized city in Yangtze River Delta, China. Environmental Geology, v. 52, n. 7, p. 1423-1433, 2007.

\section{Monte Carlo Method Implementation In Probabil- istic Risk Assessment Applied To Risk Estimation By Dermal Contact With Surface Water Contami- nated By Benzene}

\section{ABSTRACT}

Hydraulic conductivity is the most important hydrogeologic parameter, with a strong influence on the flux and contaminant transport processes in ground water. In order to verify the influences of this parameter on health risk estimation, the Probabilistic Risk Assessment process was applied to a real study case utilizing the stochastic method most widely used in science, the Monte Carlo method. For this purpose, the pathway of dermal contact exposure to surface water during swimming was considered and the stochastic risk was evaluated. The stochastic analysis results revealed a difference of six orders of magnitude between the minimum and maximum estimated values and two orders of magnitude for the real risk value, indicating the dimension of the uncertainties. The model sensibility analysis demonstrated that contaminant concentration is the parameter of highest correlation with risk, revealing the influence of hydraulic conductivity on the stochastic model uncertainties.

Key-words: Monte Carlo method, probabilistic risk assessment, stochastic risk estimation. 